\title{
Echelle-Diagrams for roAp Stars
}

\author{
David E. Mkrtichian \\ Astronomical Observatory, Odessa State University, T. G. Shevchenko \\ Park, 65014 Odessa, Ukraine \\ Artie P. Hatzes \\ McDonald Observatory, University of Texas at Austin, Austin, TX \\ 78712, USA
}

\begin{abstract}
We present the echelle-diagrams for p-mode spectra of roAp stars HD 60435, HR 1217 and $\gamma$ Equ and discuss some their peculiarities.
\end{abstract}

The high-order nonradial pulsation p-mode spacing in main sequence stars as a function of oscillation frequency is a sensitive indicator for the study of stellar structure and the location depth of convective zone (Guenther \& Demarque 1993, 1996). The frequency spectra of high-order p modes in roAp stars can well extend to higher $(\ell>3)$ degrees, and these can be detectable spectroscopically if they are indeed excited (Mkrtichian 1994). So, the diagnostic potential of the echelle-diagrams (ED) for the p-mode rich roAp stars is expected to be high.

In the roAp star HD 60435 , the frequency range $(709-1457 \mu \mathrm{Hz})$ of excited modes and their number $(n=19)$ is the widest and most numerous yet detected in roAp stars (Matthews, Kurtz, \& Wehlau 1987). As such, the star is well suited for the application of frequency spacing analysis. In the ED for HD 60435 shown in Fig. 1 (left), the modes of even and odd degrees form approximately 'equidistant' frequency spaced columns. There are probable signatures of the mode-bumping effect (for instance, the modes $1.2848 \mathrm{mHz}$ and $1.1133 \mathrm{mHz}$ shown by circles) predicted by theory (Guenther \& Demarque 1993, 1996).

The ED for the multiperiodic roAp star HR 1217 is shown in Fig. 1 (right, top). The interesting signature in the mode spectrum of this multiperiodic roAp star is the non-equidistant spacing of the $\nu_{6}$ mode (Kurtz et al. 1989; Hatzes, Kanaan, \& Mkrtichian 1999). A possible explanation of this peculiarity could be the relation of $\nu_{6}$ to higher $\ell>3$ degree modes which are shifted in spacing relative the $\ell=0,2$ and $\ell=1,3$ spacing columns.

The roAp star $\gamma$ Equ so far has 4 known excited modes (Martinez et al. 1996). The ED is shown in Fig. 1 (right, bottom). Mode discrimination was carried out using the method of "specific phases" (Mkrtichian et al., in preparation). $\gamma$ Equ, HD 60435, and HR 1217 are promising candidates for multisite high-resolution spectral campaigns using precise stellar radial velocity measurements to search for new pulsation modes (Kanaan \& Hatzes 1998; Hatzes et al. 1999).

Acknowledgments. This work was supported by CRDF grant UP2-317. 

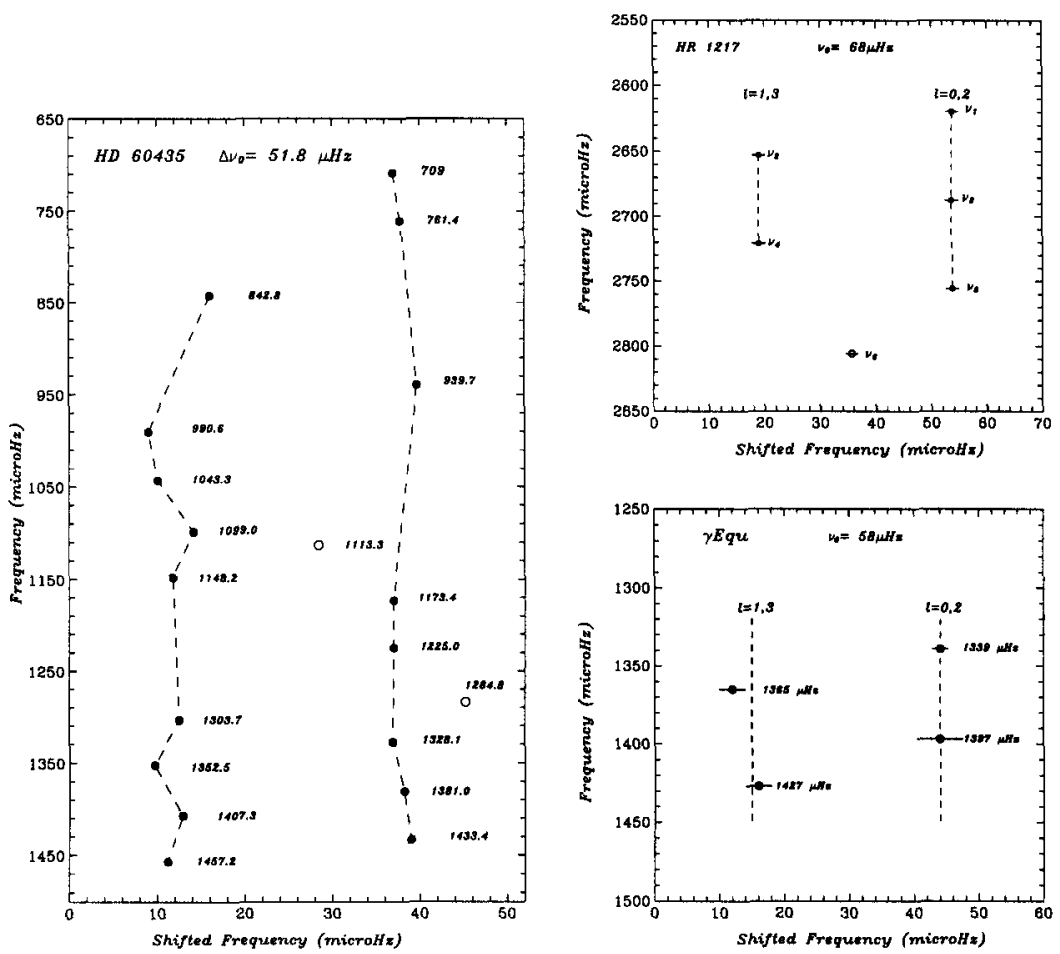

Figure 1. The echelle-diagrams for roAp stars: HD 60435 (left), $\gamma$ Equ (right, bottom) and HR 1217 (right, top).

\section{References}

Guenther, D. B. \& Demarque, P. 1993, ApJ, 405, 298

Guenther, D. B. \& Demarque, P. 1996, ApJ, 456, 798

Hatzes, A. P., Kanaan, A., \& Mkrtichian, D. E. 1999, in ASP Conf Ser. Vol. 185, Precise Stellar Radial Velocities, ed. J. B. Hearnshaw \& C. D. Scarfe (San Francisco: ASP), 166

Kanaan, A. \& Hatzes, A. P. 1998, ApJ, 27, 347

Kurtz, D. W., Matthews, J. M., Martinez, P., Seeman, J., Cropper, M., Clemens, J.C., Kreidl, T. J., Sterken, C., Schneider, H., Weiss, W. W., Kawaler, S., \& Kepler S. O. 1989, MNRAS, 240, 881

Martinez, P., Weiss, W. W., Nelson, M. J., Kreidl, T. J., Roberts, G. R., Mkrtichian, D. E., Dorokhov, N. I., Dorokhova, T. N., \& Birch, P. V. 1996, MNRAS, 282, 243

Matthews, J. M., Kurtz, D. W., \& Wehlau, W. H. 1987, ApJ, 313, 782

Mkrtichian, D. E. 1994, Solar Phys., 152, 275 